\title{
WHOLE AND PAROTID SALIVA - PROTEIN PROFILES AS SEPARATED ON 5-20\% SDS-POLYACRYLAMIDE GRADIENT GEL ELECTRO- PHORESIS AND USING MALDI-TOF MASS SPECTROMETRY
}

N.L. Huq, A. DeAngelis, Z.H.A. Rahim, M. Ung, J. Lucas, K.J. Cross, E.C. Reynolds. Whole and parotid saliva - Protein profiles as separated on 5-20\% SDSpolyacrylamide gradient gel electrophoresis and using MALDI-TOF mass spectrometry. Annal Dent Univ Malaya 2004; 11: 24-29.

\begin{abstract}
The aim was to examine the protein profiles of whole and parotid saliva using Sodium Dodecyl Sulphate Polyacrylamide Gel Electrophoresis (SDS-PAGE) and MALDI-TOF mass spectrometry. The banding patterns of proteins exhibited by the unstimulated whole saliva samples on the gel remained quite constant but the intensity of the protein bands were slightly different from one sample to another. Comparison of the protein profiles of unstimulated whole saliva and stimulated parotid saliva showed almost similar banding pattern. The exception is the presence of a pink protein band in the 65-67 kD region in the stimulated parotid saliva samples which was also observed in the unstimulated whole saliva sample contributed by a cerebral palsy patient. Analysis of the saliva samples using MALDI-TOF mass spectrometry also revealed that the stimulated parotid saliva samples exhibited some peaks that were in the same region as those for the unstimulated whole saliva sample of the cerebral palsy subject. This may imply that there is ineffective control of the parotid secretion in cerebral palsy subject under unstimulated condition. The SDS-PAGE and MALDI-TOF analyses may provide more information on the profiles of the salivary proteins which could be beneficial in the diagnosis of salivary gland dysfunction.
\end{abstract}

Key words: unstimulated whole saliva, stimulated parotid saliva, cerebral palsy, electrophoresis, mass spectrometry.

\section{INTRODUCTION}

Saliva is a glandular secretion that is vital to the maintenance of healthy oral tissues. It is constituted by the secretions of the major salivary glands, the minor salivary glands and the gingival fluid (1). Saliva keeps the oral mucosa and teeth moist and is required for lubrication, swallowing of food, mineralisation of teeth, maintenance of the oral microorganisms and many other functions $(2,3)$. Due to its constant interaction with the oral tissues, saliva

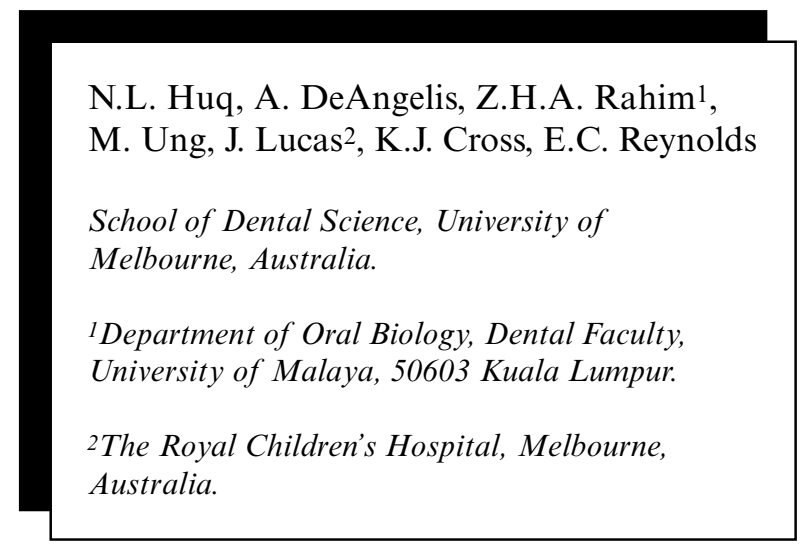

has been considered to be the major determinant of the environment within the oral cavity (4)

Whole saliva is a mixture of secretions from parotid, submandibular, sublingual and minor salivary glands mixed with bacteria, leucocytes, sloughed epithelial cells and crevicular fluids. Individual gland saliva refers to secretion from individual gland; parotid saliva from parotid gland, submandibular saliva from submandibular gland, sublingual saliva from sublingual gland. In unstimulated condition, the submandibular gland is the main contributor of the whole saliva ( $65 \%$ of the total volume) while the parotid gland contributes much less $(20 \%$ of the total volume). The parotid gland becomes the dominant gland in stimulated condition, contributing about $50 \%$ of the whole saliva (1). There were many techniques for assessing salivation and salivary secretion rate that have been reviewed and evaluated (5-8). It has been reported that whole saliva samples measurement may be superior and more relevant than individual gland secretion (5) and is said to be of relevance in the evaluation of gland dysfunction.

Saliva can be collected under unstimulated and stimulated conditions. Under stimulated condition the salivary glands are influenced by stimulants which in experimental procedures, citric acid is sometimes used as a stimulant (6). It is important to standardise the condition used for saliva collection. Accurate measures of salivary flow rate are required for a variety of clinical and experimental situations. Two methods which are commonly employed include measurement of whole saliva and measurement of parotid gland secretion rate.

There are numerous proteins present in saliva and many are multifunctional. They consist of low molecular weight proteins, such as histatin, statherin and proline-rich proteins (PRPs) and larger proteins such as cystatin and mucin. Histatin and acidic 
proline-rich proteins are the only salivary proteins found solely in saliva, whereas other salivary proteins are also present in several different body fluids (4). Statherin and PRPs are especially important in maintaining the mineralisation of tooth enamel. Histatin, cystatin and mucin have roles in the defence against resident microflora and invading microorganisms. Salivary proteins are extremely important in the homeostasis of the oral tissues and protection from oral disease, however their exact mechanisms of activity remain unclear. Separation and identification of proteins in saliva will allow for more comprehensive analyses and would lead to a better understanding of the structure and function of salivary proteins and ultimately their roles in oral diseases. In this study, the SDS-PAGE technique was used to separate the proteins based on the molecular weight and the ionic charges and the MALDI-TOF mass spectrometry was used to confirm the presence of low molecular weight proteins e.g. statherins and histatins and small peptides of proline-rich proteins. Emphasising on the identification of protein profiles that are distinct to whole and parotid saliva can be beneficial in the diagnosis of salivary glands dysfunction.

\section{MATERIALS AND METHODS}

Unless otherwise specified, all chemicals, solvents and materials used in these experiments were of analytical grade. 2,5-dihydroxybenzoic acid (DHB) and dithiothreitol (DTT) were obtained from Sigma Chemical Co. (St Louis, MO, USA). Coomassie Blue (CBB) R-250 was obtained from Bio-Rad Laboratories (Richmond, Ca, USA). Low (LMW) and High (HMW) molecular weight markers were from Amersham Pharmacia Biotechnology (Buckinghamshire, England). Acetonitrile $\left(\mathrm{CH}_{3} \mathrm{CN}\right)$, ethanol, acetic acid and sodium-dodecyl sulphate were purchased from BDH Laboratory Supplies (Poole, England). Trifluoroacetic acid (TFA) was bought from Applied biosystems (CA, USA) whilst formic acid (FA) was from Ajax Laboratory Chemicals (Auburn, NSW, Australia). Pre-cast polyacrylamide $4-20 \%$ linear gradient gels were obtained from Gradipore (North Ryde, NSW, Australia).

\section{Collection and storage of saliva samples}

Full informed consent was obtained from the donors of the samples or their legal guardians.

\section{Collection of unstimulated whole saliva}

Unstimulated whole saliva samples were collected from seven subjects whose age ranged from 14 - 40 years old. One of the subjects who drools is a confirmed cerebral palsy patient. Collection of samples were carried out not less than one hour after food consumption. Subjects were asked to rinse their mouth with tap water and collect their saliva at least 10 minutes after the rinsing. The volume of saliva collected and the time taken to collect the samples were recorded.

\section{Collection of Parotid Saliva}

Stimulated parotid saliva samples were collected through the use of a custom-made Lashley Cup. Out of the seven subjects, two volunteered to contribute their stimulated parotid saliva samples. A drop of lemon juice was applied to the tongue of the subjects at 5-10 minute intervals to stimulate parotid saliva secretion. Samples collected were stored at $-70^{\circ} \mathrm{C}$ until further analysis.

As a part of the collection procedure, subjects were required to undergo a full plaque indexing and oral examination (excluding periodontal probing). Only subjects with good oral hygiene were selected for the study.

\section{SDS-PAGE}

Samples were prepared in the following manner. $20 \mu \mathrm{L}$ of sample loading buffer $(0.125 \mathrm{M}$ tris, $4 \%$ (w/v) SDS, $0.05(\mathrm{w} / \mathrm{v})$ bromophenol blue, 20\% (v/v) glycerol) and $2 \mu \mathrm{L}$ of $1 \mathrm{M}$ DTT were added to $20 \mu \mathrm{L}$ of saliva and placed in a small eppendorf. The resulting mixture was then centrifuged for 5 seconds at $10,000 \mathrm{xg}$, heated in a dry block heater for 5 minutes and then centrifuged for another 5 seconds. SDS-PAGE was performed on a Novex Xcell II ${ }^{\mathrm{TM}}$ Mini Cell and prepared samples were loaded in 40 $\mu \mathrm{L}$ aliquots onto $4-20 \%$ linear gradient gel along with high and low molecular weight standards. Low molecular weight standards were prepared in the same manner as the saliva samples whilst the high molecular weight standards were added with no prior preparation. Samples were then run for approximately 90 minutes at a constant voltage of $150 \mathrm{~V}$ in a SDS running buffer solution $(0.1 \%(\mathrm{w} / \mathrm{v})$ SDS, $25 \mathrm{mM}$ Tris, $250 \mathrm{mM}$ glycine (electrophoresis grade), $\mathrm{pH} 8.3$ ). Upon completion of the above procedure, the gel was placed immediately in a $0.1 \%$ Coomassie brilliant blue R-250 staining solution (CBB R-250 (0.1\% (w/v) CBB R-250, 40\% (v/v) ethanol, 10\% (v/v) acetic acid) and allowed to stain for at least 3 hours. The gels were then destained for several days in a $10 \%$ acetic acid solution for optimal resolution of the pink staining PRPs.

\section{MALDI-TOF-Mass Spectrometry}

Saliva $(10 \mu \mathrm{L})$ was diluted with $10 \mu \mathrm{L}$ of a standard solution (66\% Milli-Q water, 33\% acetonitrile, $1 \%$ formic acid). The samples were then vortexed for 15 seconds and sonicated for 5 minutes. The matrix solution used consisted of a saturated solution of DHB (gentisic acid or 2,5Dihydroxybenzoic acid) in 66\% Milli-Q, 33\% acetonitrile and $1 \%$ formic acid. The matrix solution was vortexed for 10 seconds centrifuged for 10 seconds at 10,000 g. Analysis was performed on a 
PerSeptive Biosystems Voyager DE MALDI-TOF Mass Spectrometer in linear mode calibrated using Angiotensin II and ACTH residues 18-39 as standards. Samples were loaded onto sample plates as follows. $0.3 \mu \mathrm{L}$ of the matrix solution was aliquoted onto the sample plate and then immediately after, $0.3 \mu \mathrm{L}$ of the prepared saliva solution was aliquoted on top of the matrix. The samples were then air-dried before being placed within the instrument. Data was collected in positive ion mode with a voltage of $20 \mathrm{kV}$ and a delay time of $0.1 \mu \mathrm{s}$. The laser intensity was varied to optimise the resolution of the spectrum. The procedure was controlled using Voyager-DE Bio-spectrometry Workstation 4.03 PC software whilst data was visualised using PerSeptive GRAMS 3.03 software.

\section{RESULTS AND DISCUSSION}

\section{SDS-PAGE of Saliva Samples}

SDS-PAGE was used to provide a comparison of the protein profiles of whole unstimulated and stimulated parotid saliva (Figure 1). Proteins were identified using electrophoretic data from a previous study (9) as well as by comparison to molecular weight markers using the known molecular weight of certain proteins. The pink stained bands, resulting from metachromasia of CBB R-250 dye in the presence of closely spaced proline residues, correspond to the various proline-rich proteins (PRPs) found in saliva (9). The dark violet band just below the $94 \mathrm{kDa}$ marker is expected to be lactoferrin $(90$ $\mathrm{kDa}$ ) is present in all of the whole unstimulated and stimulated parotid saliva samples. The next violet band near the $67 \mathrm{kDa}$ is thought to correspond to albumin (66 kDa) appeared to be absent in the parotid saliva. The broad violet doublet just below the $67 \mathrm{kDa}$ marker can be identified as the glycosylated and non-glycosylated forms of $\alpha$ amylase. A pink band may be present above this doublet, as seen in lane 7 and X (Figure 1), and is thought to be a glycosylated basic PRP (9). The bands found in the 30-43 kDa region most probably contain other basic PRPs whilst the very prominent pink and blue bands between 30 and $20.1 \mathrm{kDa}$ correspond to the acidic PRPs (9). Those below the $20.1 \mathrm{kDa}$ marker are likely to be a mixture of basic PRPs, histatins, statherin and cystatins, purely based on their molecular weights.

In most respects, the banding patterns of proteins exhibited by these saliva samples remained quite constant from one individual to another (Figure 1). Most major differences could be attributed to slight differences in concentration of the various proteins between one sample and the next. Comparison of the stimulated parotid saliva and the unstimulated whole saliva from cerebral palsy subject showed a similarity in one of the protein profiles which is not seen in other unstimulated whole saliva.

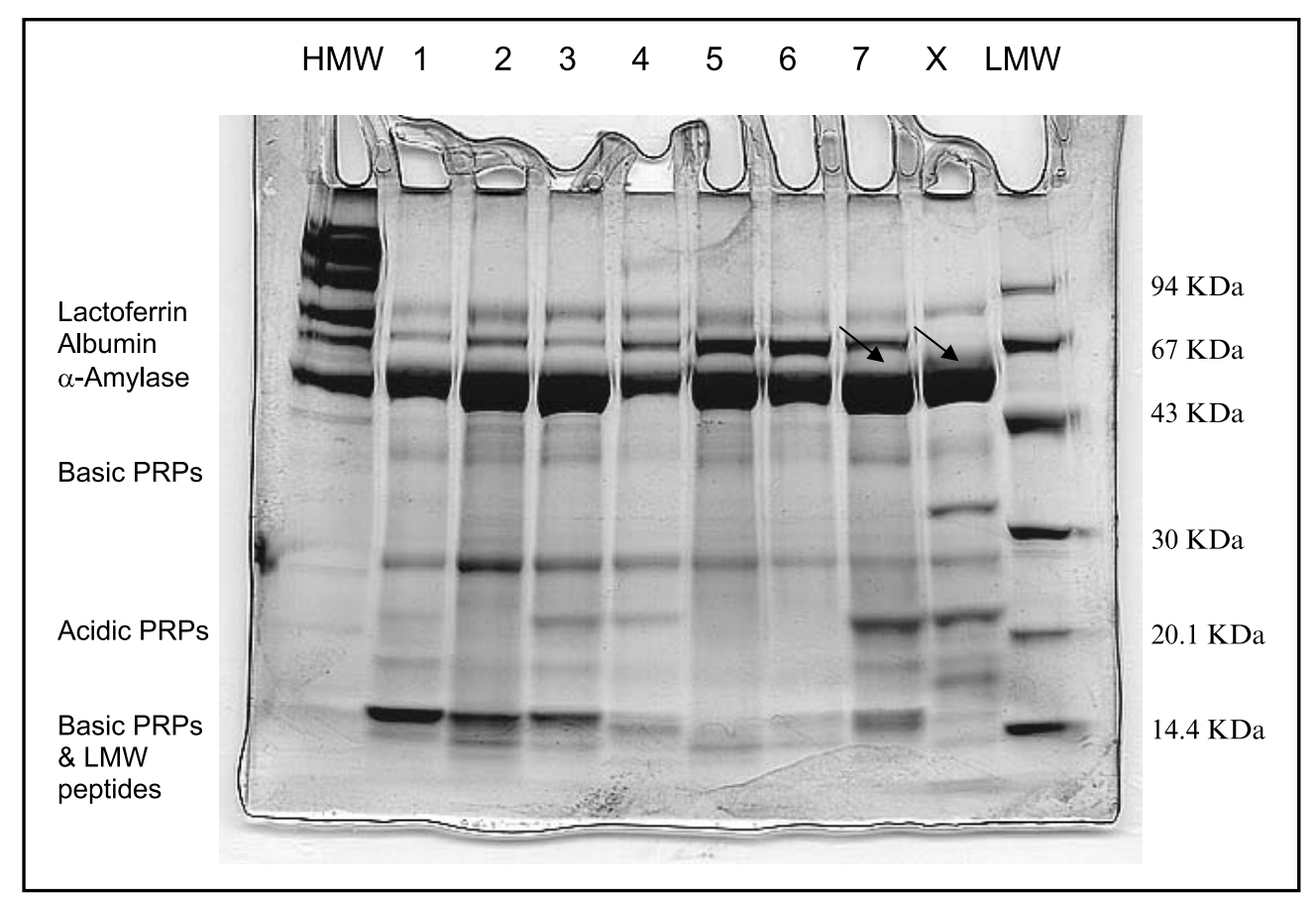

Figure 1: SDS Gradient Gel showing protein profiles of unstimulated whole saliva of healthy subjects (Lanes 1,2,3,4,5 and 6); cerebral palsy patient (Lane 7) and stimulated parotid saliva (Lane X). HMW refers to high molecular weight protein standards and LMW refers to low molecular protein standards. The arrows showed the additional bands seen in unstimulated whole saliva from cerebral palsy subject and stimulated parotid samples of healthy subjects. 


\section{MALDI- TOF-MS analysis of saliva}

Figures 2, 3 and 4 show the protein profiles of the saliva samples as analysed by mass spectrometry. Generally all of the protein profiles in unstimulated whole saliva looked remarkably similar with groups of peaks occurring around the $\mathrm{m} / \mathrm{z} 1350,1860,2900$, 3373, and 3444 and $3490 \mathrm{~m} / \mathrm{z}$ marks. A small set of peaks may also be seen at the $5300 \mathrm{~m} / \mathrm{z}$ mark. The triplet of peaks at m/z 3373, 3444 and 3490 Da were observed in all the unstimulated whole saliva samples, and matched the known molecular weights of the human neutrophil $\alpha$-defensins 2, 1 and 3, respectively (Figure 2 and 3 ). The $\alpha$-defensins are produced by neutrophil cells and are part of the defensin protein family, which are known to possess a wide range of antimicrobial functions. $\alpha$-defensins 1, 2 and 3 have been shown to be overexpressed in squamous cell carcinoma tissue(10) and significantly increased in the saliva derived from patients with oral squamous cell carcinomas (11)

The peaks (m/z 3373, 3444 and 3490) that were found in whole unstimulated saliva are absent in stimulated parotid saliva. However, the peaks $(\mathrm{m} / \mathrm{z}$
4373, 4930 and 5388) exhibited by the unstimulated whole saliva of cerebral palsy patient were observed to be present in the stimulated parotid saliva (Figure 4). This is an interesting observation which imply that in cerebral palsy patient, the parotid secretion is predominant. Previously, it has been reported that the submandibular glands contribute more to the whole saliva in unstimulated condition $(1,12,13)$. The drooling effect observed in cerebral palsy may be due to ineffective control of the parotid gland secretion.

\section{CONCLUSION}

From the SDS-PAGE, the protein profiles of saliva from various subjects were found to be similar but varied in the electrophoretic band intensities, reflecting differences in protein concentrations. The parotid saliva also revealed a greater number of pink PRP proteins compared to whole saliva, as indicated by the presence or lack of pink staining bands. From the MALDI-TOF mass spectrometry and

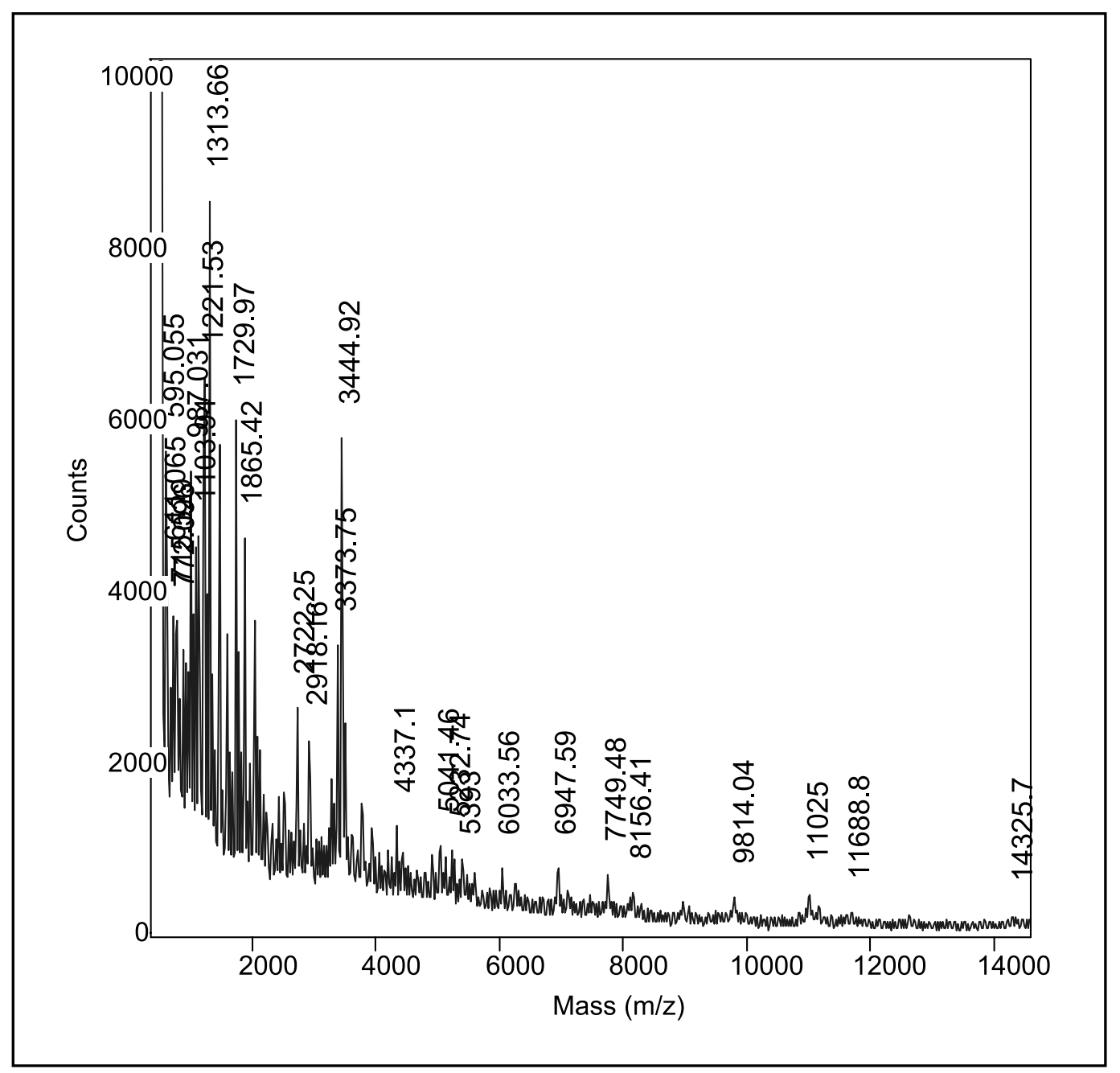

Figure 2: Mass spectrometry profiles of the protein components representing the unstimulated whole saliva. 


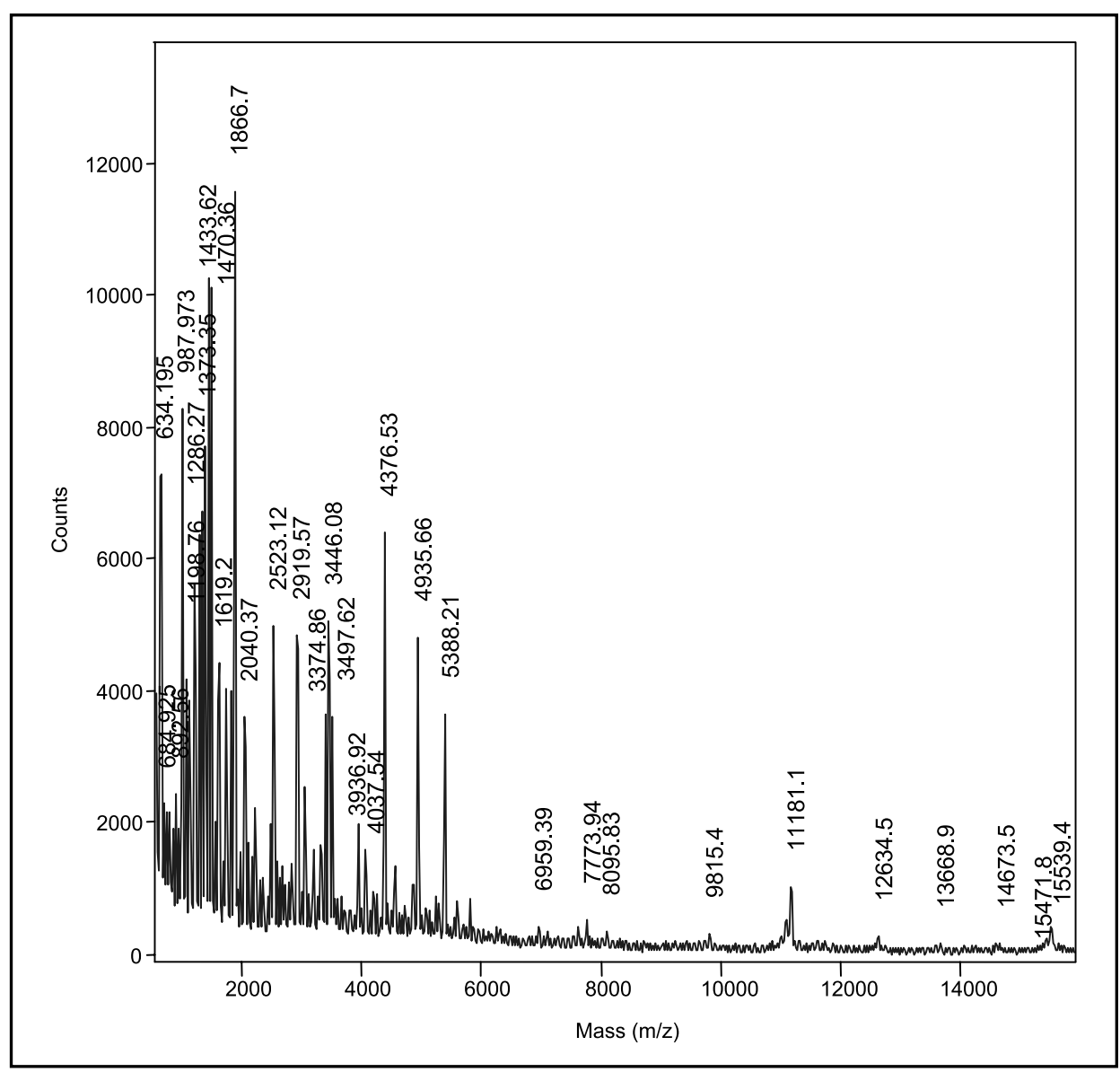

Figure 3: Mass spectrometry profiles of the protein components in the unstimulated whole saliva sample from cerebral palsy subject.

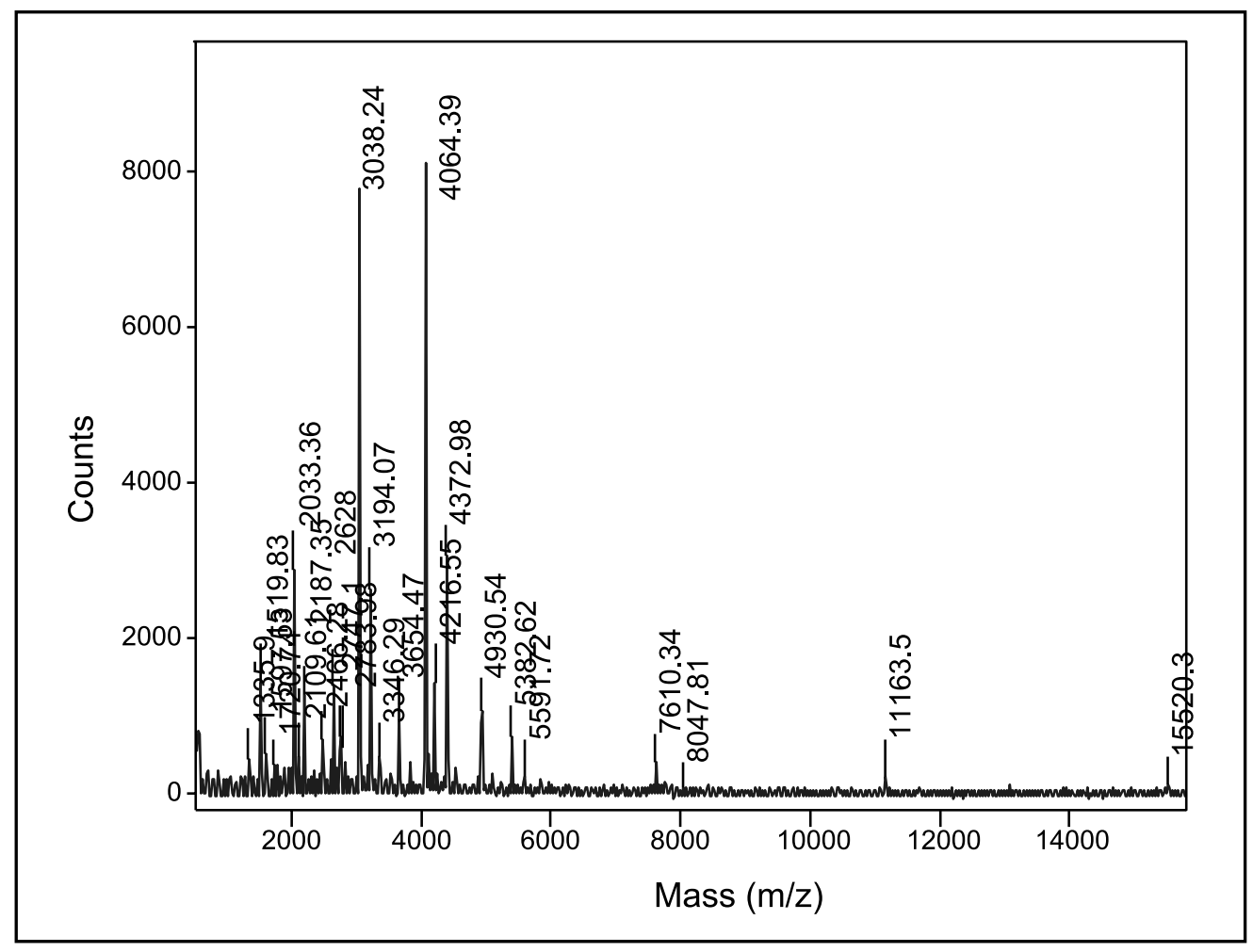

Figure 4: Mass spectrometry profiles of the protein components in the stimulated parotid saliva sample from normal subjects. 
comparison of the mass spectra of all the saliva samples showed: a) an overall similarity between the various unstimulated whole saliva; b) some protein peaks observed to be present in unstimulated whole saliva sample were absent in the stimulated parotid saliva and c) the unstimulated whole saliva from cerebral palsy subjects exhibited some protein peaks in the same region as those of the stimulated parotid saliva. In conclusion, more information on the salivary protein profiles can be derived using SDSPAGE and MALDI-TOF mass spectrometry.

\section{REFERENCES}

1. Edgar WM, O’Mullane DM. Saliva and oral health. British Dental Association, 1996. $2^{\text {nd }}$ Edition. London.

2. Johnson DA, Yeh CK, Dodds MWJ. Effect of donor age on the concentrations of histatins in human parotid and submandibular/sublingual saliva. Arch Oral Biol; 2000; 45: 731-40.

3. Johnsson M, Richardson CF, Bergey EJ, Levine MJ, Nancollas GH. The effects of human salivary cystatins and statherins on hydroxyapatite crystallization. Arch Oral Biol; 1991; 36: 631-6.

4. McNamara TF, Friedman BK, Roth P. Salivary access as an ecological determinant. In proceedings: Saliva and dental caries, Kelinberg I, Ellison SA and Mandel ID. Eds, 1979; Information Retrieval, New york, 211.

5. Navazesh M, Christensen CM. A comparison of whole mouth resting and simulated salivary measurement procedures. J Dent Res; 1982; 61: 1158.
6. Kerr AC. The physiological regulation of salivary secretions in man. A study of the response of human salivary glands to reflex stimulation. International Series of Monographs on Oral Biology; 1961. Vol 1.

7. Mason DK, Chisholm DM. Salivary glands in health and disease. WB Saunders, London; 1975.

8. White KD. Salivation: a review and experimental investigation of major techniques, Physiology; 1977; 14: 203.

9. Beely J, Sweeney D, Lindsay J, Buchanan M, Sarna L, Khoo KS. Sodium dodecyl sulphatepolyacrylamide gel electrophoresis of human parotid salivary proteins. Electrophoresis; 1991; 12: $1032-41$.

10. Lundy, FT, Orr DF, Gallagher JR, Maxwell P, Shaw C, Napier SS, Gerald Cowan C, Lamey PJ, Marley JJ. Identification and overexpression of human neutrophil alpha-defensins (human neutrophil peptides 1, 2 and 3 ) in squamous cell carcinomas of the human tongue. Oral Oncol; 2004; 40(2): 139-44.

11. Mizukawa N, Sugiyama K, Fukunaga J, Ueno T, Mishima K, Takagi S, Sugahara T. Defensin1 , a peptide detected in the saliva of oral squamous cell carcinoma patients. Anticancer Res; 1998; 18(6B): 4645-9.

12. Humphrey SP, Williamson RT. A review of saliva: normal composition, flow, and function. J Prosthet Dent; 2001; 85(2): 162-9.

13. Ferguson DB. The salivary glands and their secretions. In Oral Bioscience. Churchill Livingstone@ Harcourt Publishers limited 1999; pg 147. 